\title{
G-Series Nerve Agent GC
}

National Cancer Institute

\section{Source}

National Cancer Institute. G-Series Nerve Agent GC. NCI Thesaurus. Code C161527.

A highly toxic chemical precursor of the nerve agent sarin, a rapid-acting non-persistent org anophosphorus ester that is a clear, colorless and odorless liquid and is absorbed into the body by inhalation, ingestion, skin contact, or eye contact, and causes excessive cholinergic stimulation by producing potent and irreversible inhibition of cholinesterase. The effects of GC are similar but less severe than sarin. 